\title{
Precision Cosmology from large-scale structure observations
}

\author{
Ariel Sánchez* \\ Max-Planck-Institut für extraterrestrische Physik, Postfach 1312, Giessenbachstr., 85741 \\ Garching, Germany. \\ E-mail: arielsan@mpe.mpg.de
}

The discovery of the accelerated expansion of the Universe has revolutionized the field of physical cosmology. Understanding the origin of this phenomenon is one of the most outstanding problems in physic today. Observations of the large-scale structure (LSS) of the Universe are expected to play a mayor role at shedding light on this problem. Driven by the potentiality of these observations, several new ground-breaking galaxy surveys are currently under way. These surveys will measure the LSS of the Universe with unprecedented precision, providing new insights not only on the origin of cosmic acceleration, but also on many other important physical parameters. Here we review the cosmological information encoded in the LSS of the Universe and the advantages and limitations of the methods most commonly used to retrieve it. As an illustration, we describe the cosmological implications of the large-scale galaxy clustering in the baryon oscillation spectroscopic survey (BOSS), with an emphasis on the problem of cosmic acceleration.

VIII International Workshop on the Dark Side of the Universe

June 10-15, 2012

Buzios, Rio de Janeiro, Brasil

* Speaker. 


\section{Introduction}

One of the most significant scientific discoveries of recent years is that, not only is our Universe expanding, it is doing so at an increasing rate [1], 2]. Understanding is the origin of the current phase of accelerated expansion is one of the most important unsolved problems in physics today, as it may have far reaching implications for our knowledge of the fundamental laws of nature. Within the context of homogeneous and isotropic solutions to Einstein's general relativity, the attractive force of gravity due to all know constituents of the Universe should lead to a deceleration of the expansion. Then, the observed acceleration implies that the total energy-density of the Universe is dominated by an exotic component, dubbed dark energy, whose repulsive effect drives the acceleration of cosmic expansion. Although current observations allow us to detect the presence of this component, its true nature still eludes our understanding. A key property that can be used to characterize it is the dark energy equation of state, $w_{\mathrm{DE}}=p_{\mathrm{DE}} / \rho_{\mathrm{DE}}$, where $p_{\mathrm{DE}}$ and $\rho_{\mathrm{DE}}$ correspond to the pressure and density of dark energy, respectively. Current observations are consistent with the so-called $\Lambda \mathrm{CDM}$ cosmological model, in which the dark energy component can be characterized by a constant equation of state $w_{\mathrm{DE}}=-1$ which can be associated with the quantum energy of the vacuum or Einstein's cosmological constant. A detection of a deviation from $w_{\mathrm{DE}}=-1$, at any time in cosmic history, would have strong implications on our understanding of cosmic acceleration.

The first step into solving the mystery of cosmic acceleration is to obtain accurate measurements of the expansion history of the Universe. Observations of the large-scale galaxy clustering can provide us with such measurements. Galaxies form a variety of structures which encode valuable information about our Universe. Observations of this clustering pattern have played a central role in establishing the current standard cosmological model [3]-6], and it is expected that they will continue to do so in the coming years. The information provided by observations of the large-scale structure (LSS) of the Universe is highly complementary to that of cosmic microwave background (CMB) measurements, as it helps to break degeneracies between certain parameter combinations which are inherent to this dataset 仍. The combination of CMB and LSS datasets has been used to place tight constraints on the basic set of cosmological parameters, restricting the range of possible deviations from the of the $\Lambda$ CDM model [8, 10, 11, 9, 12, 15].

Over the past decades the size and quality of galaxy redshift surveys has increased dramatically. The pioneering efforts of the CfA Redshift Survey [16] and the Las Campanas Redshift Survey (LCRS) [17], which provided the first clear three-dimensional view of the galaxy distribution in the local Universe, were followed by several other surveys covering different volumes. Among them the two-degree Field Galaxy Redshift Survey (2dFGRS) [18] and the Sloan Digital Sky Survey (SDSS) [19] have played a pivotal role at mapping the large-scale galaxy distribution, providing the first accurate large-scale galaxy clustering measurements and marking the start of a data-rich era in cosmology.

Driven by the potential of LSS observations for shedding light on the nature of dark energy, the tendency to construct increasingly larger surveys will continue in the coming years. A new generation of galaxy surveys is currently being constructed or designed. Examples of these new surveys include the completed WiggleZ [20], the ongoing Baryon Oscillation Spectroscopic Survey (BOSS) [21], and future surveys like the ESA space mission Euclid [22]. By probing larger volumes, these surveys can provide more accurate views of the large-scale galaxy clustering pattern 
than ever before, offering an opportunity to obtain new constraints not only on dark energy, but also neutrino masses, the curvature of the Universe or inflationary physics.

This revolution in the observational data poses a challenge for our theoretical understanding and modelling of LSS observations, which is in danger of lagging behind these new advances. The increase in the amount and quality of the observational data in the coming years will lead to a drastic reduction of the statistical errors of LSS measurements. In this context, the control of the systematic errors introduced by our analysis techniques becomes crucial as these might become dominant in the final error budget. LSS observations are affected by scale-dependent effects which, if not taken into account correctly, might jeopardise their use as precision cosmological probes. Fortunately, new theoretical tools have been developed over the past few years that might hold the key to extracting unbiased cosmological information from LSS datasets. In view of these rapid improvements in the observational side, it is timely to review these recent advances.

Here we review the basic aspects of the statistical analysis of large-scale structure observations, and the results that present day galaxy samples can provide. In Section 2 we describe the most commonly used statistical tools to analyse galaxy surveys, the two-point correlation function and the power spectrum. In Section 3 we analyse the potential systematics that could affect LSS measurements as well as the models that can be used to describe them or to minimise their impact. In Section $\emptyset$ we use recent results from BOSS to illustrate the cosmological constraints achievable with present day galaxy catalogues. Finally, Section 5 contains our main conclusions.

\section{Statistical analysis of large-scale structure observations}

The most commonly used tools to extract cosmological information out of galaxy redshift surveys are two-point statistics such as the correlation function, $\xi(s)$, and the power spectrum, $P(k)$. These are defined in terms of the fluctuations in the density field

$$
\delta(\mathbf{x})=\frac{n(\mathbf{x})-\bar{n}}{\bar{n}}
$$

where $n(\mathbf{x})$ represents the density at a given location $\mathbf{x}$ and $\bar{n}$ corresponds to the mean density field. The two-point correlation function is given by

$$
\xi(\mathbf{r})=\langle\delta(\mathbf{x}) \delta(\mathbf{x}+\mathbf{r})\rangle,
$$

where the brackets represent an ensemble average. The hypothesis of homogeneity and isotropy of the density field imply that the correlation function must depend only on the modulus of the separation vector $r=|\mathbf{r}|$

Another commonly used tool is the galaxy power spectrum, $P(k)$, defined in an analogous way as the two-point correlation function, but in terms of the Fourier transform of the density fluctuations, $\tilde{\delta}(k)$, as

$$
\left\langle\delta \tilde{(\mathbf{k})} \delta\left(\tilde{\mathbf{k}}^{\prime}\right)\right\rangle=(2 \pi)^{3} \delta_{D}\left(\mathbf{k}-\mathbf{k}^{\prime}\right) P(k),
$$

where $\delta_{D}(\mathbf{k})$ is the usual Dirac delta function. It is easy to show that the power spectrum and the two-point correlation function are a Fourier transform pair, with

$$
\xi(r)=\frac{1}{2 \pi^{2}} \int P(k) j_{0}(k r) k^{2} \mathrm{~d} k
$$



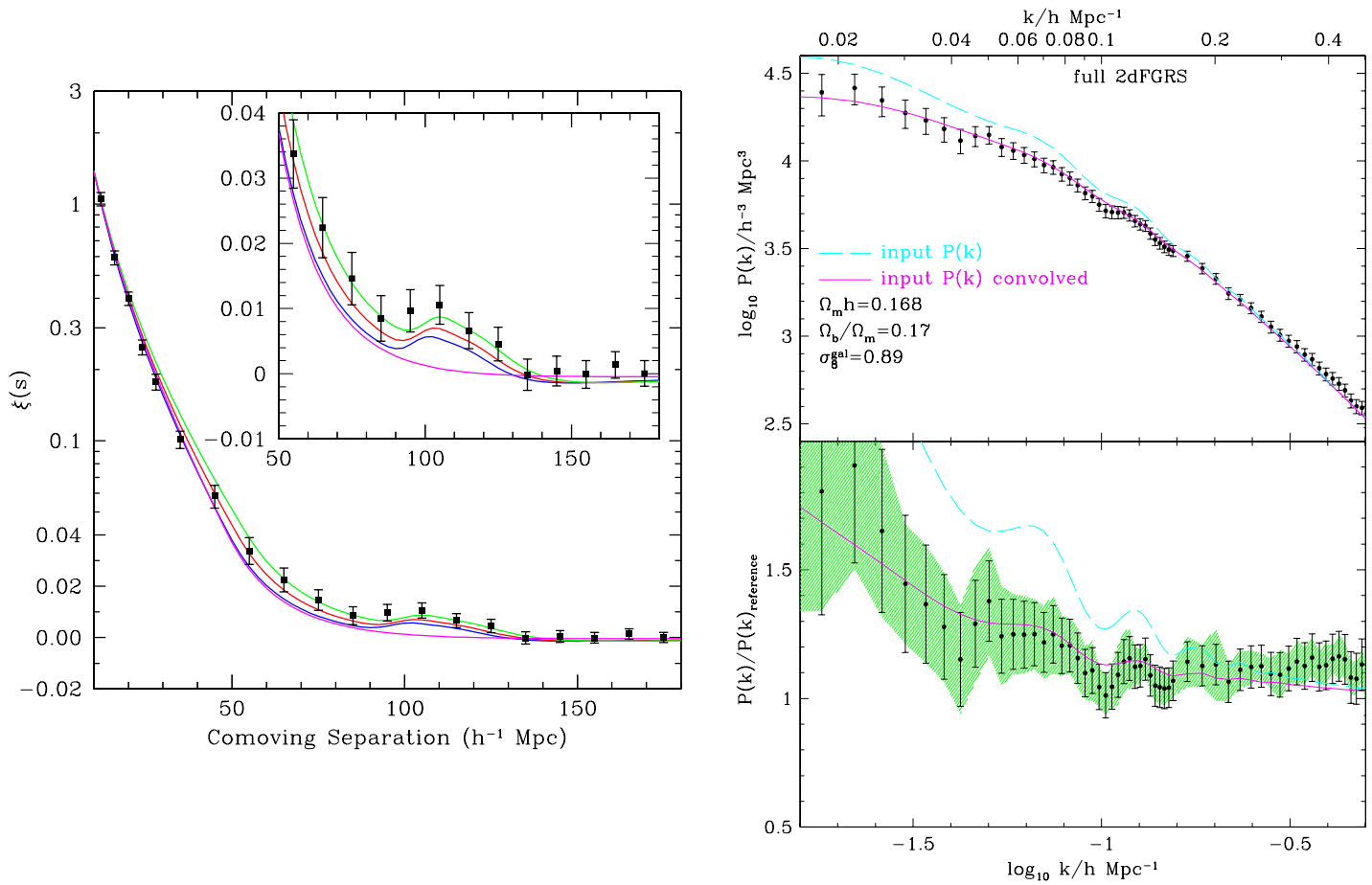

Figure 1: Left: the large-scale correlation function of the LRG sample from SDSS DR3 []. The BAO signal appears as a broad peak on large-scales which can be seen more clearly in the inset. Figure from Eisenstein et al. [6]. Right: the upper panel shows the power spectrum of the final 2dFGRS. The BAO signal can be seen more clearly in the lower panel, which shows the ratio of this measurement to a reference function with the same overall shape but no oscillations. Figure from Cole et al. [5].

where $j_{0}(x)$ is the spherical Bessel function of order zero.

The left-had panel of Fig. 1 shows the measurement of the large-scale correlation function of the Luminous Red Galaxy (LRG) sample from the SDSS data release 3 (DR3) [6]. The right-hand panel of the same figure shows the power spectrum of the final 2dFGRS [5]. The full shape of these functions encode useful cosmological information. Measurements of these statistics from various galaxy samples have been used, in combination with other datasets, to obtain constraints on the values of the basic cosmological parameters [5, 6, 8- 15.

A special feature of the large-scale galaxy clustering provides a powerful method to probe the expansion history of the Universe: the signature of the baryon acoustic oscillations (BAO). These are fluctuations in the density field caused by acoustic waves which propagated through the photonbaryon fluid prior to recombination. The signature of the BAO appears as a broad peak in the large scale correlation function [23], which can be clearly seen in the inset plot of the left-hand side of Fig. 1. In the power spectrum, the BAO signal appears as a small oscillatory amplitude modulation which can be seen more clearly in the lower panel of the right-hand side of Fig. 1. which shows the ratio of the $2 \mathrm{dFGRS} P(k)$ to a reference function with the same overall shape as the measurement, but no oscillations. The measurements shown in this figure correspond to the first detections of the BAO feature in the large-scale galaxy clustering. Since then, the BAO signal has been confirmed 
with increasing precision using a variety of datasets and techniques [24, 13, 15].

The mere detection of the BAO signal in the large-scale galaxy clustering is significant, as it confirms a clear prediction of the current paradigm of the growth of structure by gravitational collapse from the small density fluctuations that can be seen in the CMB. Besides this, the BAO signal is important for more practical reasons. The position of the acoustic peak in the two-point correlation function and the wavelength of the oscillations in the power spectrum are closely related to the size of the sound horizon at the drag redshift, $r_{\mathrm{s}}\left(z_{\mathrm{d}}\right)$. As this scale, of about $150 \mathrm{Mpc}$ (comoving), can be measured with high accuracy from CMB observations [25], the BAO signal inferred from galaxy clustering measurements can be used as a standard ruler. The apparent size of the BAO ruler in the directions parallel and perpendicular to the line-of-sight can be used to measure the redshift evolution of the Hubble parameter, $H(z)$, and the angular diameter distance, $D_{\mathrm{A}}(z)[26]$. However, when dealing with angle-averaged measurements such as those shown in Fig. 1], the BAO signal is only sensitive to an average distance, $D_{\mathrm{V}}(z)$, given by [6]

$$
D_{\mathrm{V}}(z)=\left((1+z)^{2} D_{\mathrm{A}}(z)^{2} \frac{c z}{H(z)}\right)^{1 / 3} .
$$

In this way, BAO measurements from galaxy samples at different redshifts can be used to probe the redshift-distance relation. Without including the CMB information on $r_{\mathrm{s}}\left(z_{\mathrm{d}}\right)$, BAO measurements can constrain distances relative to the size of the ruler, that is, $D_{\mathrm{V}}(z) / r_{\mathrm{s}}\left(z_{\mathrm{d}}\right)$. Thanks to the BAO signal LSS observations offer one of the most promising routes to obtain accurate constraints on $w_{\mathrm{DE}}$ and its possible evolution with time.

\section{Modelling large-scale structure observations}

The quality of current and future galaxy surveys demands the use of accurate models to extract unbiased cosmological constraints. In this section we review the potential systematic effects that affect LSS observations (Section 3.1), the way in which they can be taken into account in our models (Section 3.2), as well as a technique that can reduce their impact (Section 3.3).

\subsection{Potential systematics of clustering measurements}

In order to establish the link between LSS observations and cosmological models, simplifying assumptions were usually made in which galaxy clustering was expected to closely follow the predictions of linear perturbation theory for the matter. However, thanks to new developments in perturbation theory and the analysis of large N-body simulations, it has become clear that this simple picture is not sufficiently accurate to describe current datasets [27-29]. Even on large scales, the clustering of galaxies is distorted by the non-linear growth of density fluctuations, redshiftspace distortions and a possible non-trivial relation between the distribution of galaxies and the underlying dark matter density field. We will now see how each of these effects change the shape of the large-scale clustering measurements.

The effect of non-linear evolution on large-scale clustering measurements can be seen in the blue circles in the left-hand panel of Fig. 目, which correspond to the mean $\xi(s)$ of an ensemble of large-volume N-Body simulations [30, 29]. The shaded region indicates the variance between the different realizations. The shape of the $\mathrm{BAO}$ peak strongly deviates from the predictions of linear 

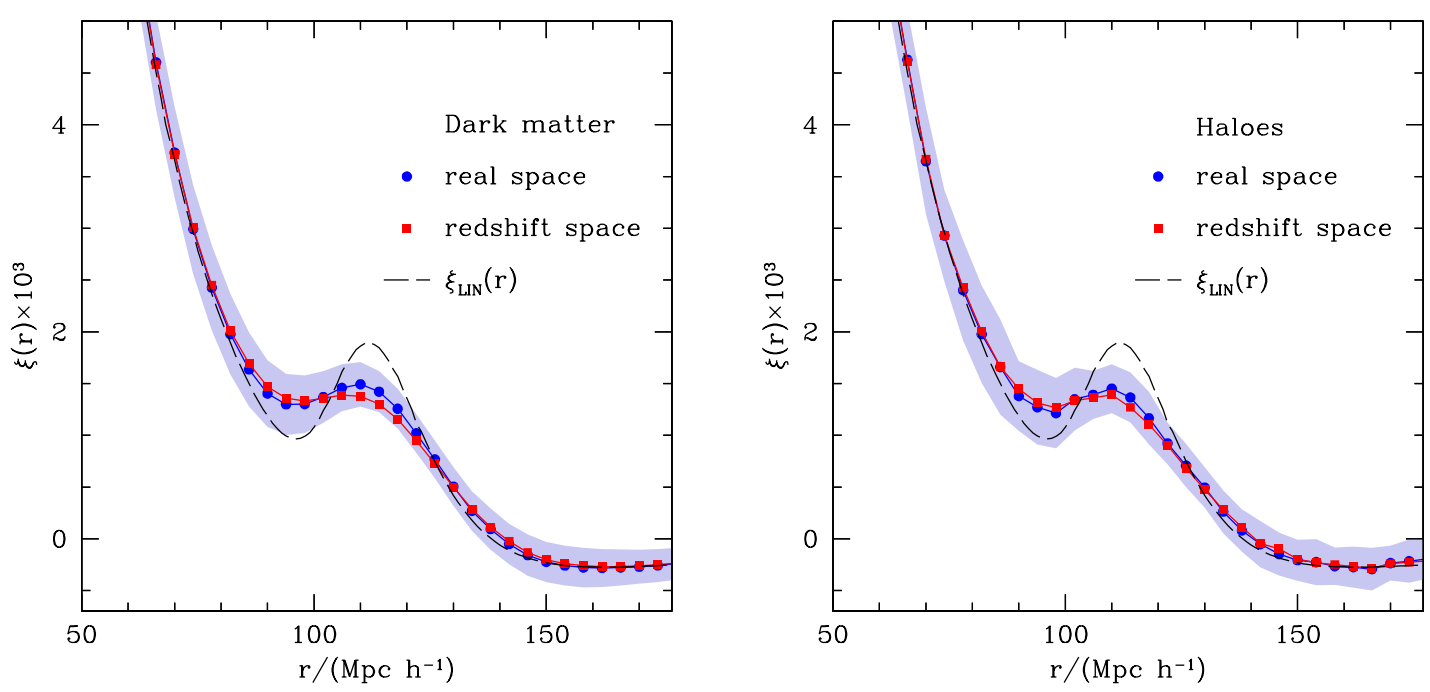

Figure 2: Left: mean large-scale matter correlation function from an ensemble of large-volume largevolume N-Body simulations [30, 29] in real- (circles) and redshift-space (squares). Right: equivalent to the left panel but for a sample of dark matter halos in the same set of simulations. The Non-linear evolution of density fluctuations and redshift-space distortions affect the shape of the BAO peak.

perturbation theory, which are shown by the dashed line. Non-linear evolution damps the acoustic peak and shifts its centroid towards smaller scales. If unaccounted for, these distortions can bias the constraints obtained from large-scale clustering measurements.

A further complication of the analysis of galaxy clustering measurements is due to the fact that the distances to individual objects are inferred from their observed redshifts, $z_{\text {obs }}$. Besides the component due to the universal expansion, these contain an extra contribution due to the peculiar velocities of the galaxies along the line of sight, $v_{\|}$, as

$$
1+z_{\mathrm{obs}}=\left(1+z_{\mathrm{cos}}\right)\left(1+z_{\text {pec }}\right),
$$

with $z_{\text {pec }}=v_{\|} / c$. This means that the inferred radial distances deviate from the true ones, distorting the observed clustering pattern. However, as the galaxy peculiar velocity field is induced by the underlying matter distribution, it is possible to model the distortions that will arise in a given cosmological model. This is a simple problem in the linear regime, where redshift-space distortions lead to a scale-independent boost of the amplitude of the power spectrum and the correlation function by a factor [31]

$$
S \equiv \frac{\xi_{\mathrm{s}}(r)}{\xi(r)}=\left(1+\frac{2}{3} f+\frac{1}{5} f^{2}\right)
$$

where $f=\mathrm{d} \ln D / \mathrm{d} \ln a$ and $D(a)$ is the growth factor of density fluctuations.

The red squares in the left-hand panel of Fig. 目correspond to the mean correlation function of the same ensemble of simulations, but measured using the redshift-space position of the particles. Here, the measurements have been rescaled by the inverse of the boost factor of equation (3.2), which correctly accounts for the change in the overall amplitude of the correlation function. There is, however, a more subtle effect of redshift-space distortions on the shape of $\xi_{\mathrm{s}}(r)$, as they lead to 
an extra damping of the BAO peak, which can be associated with the smearing of the velocity field along the line of sight due to large-scale random motions, such as the fingers-of-God effect [32].

Additionally, our observations probe the galaxy density field, which could be a modified version of the underlying matter distribution. According to the current understanding of galaxy formation, galaxies are hosted within dark matter halos. Hence, the analysis of the clustering of dark mater halos from $\mathrm{N}$-body simulations can give us a first order description of galaxy clustering. On large scales, a simple linear relation is expected to hold between the fluctuations in the density of halos of a given mass $m, \delta_{\mathrm{h}}(\mathbf{r} \mid m)$, and the matter density field, that is [33, 34]

$$
\delta_{\mathrm{h}}(\mathbf{r} \mid m)=b(m) \boldsymbol{\delta}(\mathbf{r}) .
$$

This implies that the correlation function of dark matter halos, $\xi_{\mathrm{h}}(r)$, will be related to the matter correlation function by a scale-independent effective bias factor, $b_{\mathrm{eff}}^{2}=\xi_{\mathrm{h}}(r) / \xi(r)$, which can be computed by taking the weighted average of the bias $b(m)$ over the selected halo sample as

$$
b_{\text {eff }}=\frac{\int_{0}^{\infty} \phi(m) b(m) n(m) \mathrm{d} m}{\int_{0}^{\infty} \phi(m) n(m) \mathrm{d} m},
$$

where $n(m)$ is the mass function of dark matter halos [34], and $\phi(m)$ represents the mass selection function applied to construct the halo sample. Additionally, the halo correlation function is affected by redshift-space distortions which, in the linear regime, lead to a rescaling of the amplitude of $\xi_{\mathrm{h}}(r)$ by a factor analogous to that of equation (3.2), where $f$ is replaced by the parameter $\beta=$ $f / b_{\text {eff. }}$.

The right-hand panel of Fig. Z shows the mean $\xi_{\mathrm{h}}(r)$ of a sample of halos from the same simulations in real- (blue circles) and redshift-space (red squares). The real-space halo correlation function has been rescaled to match the amplitude of the matter $\xi(r)$ using its effective bias factor. The redshift-space results have also been rescaled by the Kaiser boost factor for the same effective bias. These measurements show that the scale-dependent effects caused by non-linear evolution and redshift-space distortions depend on the particular halo sample being analysed and do not correspond exactly to those of the matter distribution, adding an extra complication to the modelling of LSS observations.

Although this discussion has been based on the two-point correlation function, the power spectrum is also affected by these scale-dependent effects in a similar way [30]. These distortions cannot be ignored when modelling LSS observations as they could lead to a significant bias in the obtained cosmological constraints.

\subsection{Modelling large-scale structure observations}

Improving our theoretical understanding of galaxy clustering has been the main focus of substantial work over recent years. The modelling of the non-linear evolution of density fluctuations has been revitalized by several new approaches to perturbation theory [35-38], which provide a better understanding of the physical processes that shape clustering measurements such as the power spectrum or the correlation function.

Within the theoretical framework of Renormalized Perturbation Theory (RPT), Crocce \& Scoccimarro [35] showed that the series expansion describing the non-linear power spectrum, 
$P_{\mathrm{NL}}(k, z)$, in standard perturbation theory can be reorganized as the sum of two contributions containing different physical information, of the form

$$
P_{\mathrm{NL}}(k, z)=P_{\mathrm{L}}(k, z) G^{2}(k, z)+P_{\mathrm{MC}}(k, z),
$$

where the redshift evolution of the linear-theory power spectrum, $P_{\mathrm{L}}(k, z)$, is given by the linear theory growth factor $D(z)$. The function $G(k, z)$, dubbed non-linear propagator, weights how much power can be directly linked to the linear epoch, while $P_{\mathrm{MC}}$ represents the power arising from the coupling of different Fourier modes. To a good approximation, the non-linear propagator is of Gaussian form,

$$
G(k, z) \simeq \exp \left(-\left(\frac{k}{\sqrt{2} k_{\star}}\right)^{2}\right),
$$

with a damping scale, $k_{\star}$, is given by

$$
k_{\star}(z)=\left[\frac{1}{6 \pi^{2}} \int \mathrm{d} k P_{\mathrm{L}}(k, z)\right]^{-1 / 2} .
$$

The mode coupling power spectrum is given by the sum of an infinite number of terms ordered by the number of initial modes coupled. At the lowest order, $P_{\mathrm{MC}}(k)$ is generated by the coupling of two modes, which at low- $k$ can be approximated by

$$
P_{1 \text { loop }}(k)=\frac{1}{2 \pi^{2}} \int \mathrm{d}^{3} q\left|F_{2}(\mathbf{k}-\mathbf{q}, \mathbf{q})\right|^{2} P_{\mathrm{L}}(|\mathbf{k}-\mathbf{q}|) P_{\mathrm{L}}(q),
$$

where $F_{2}(\mathbf{k}, \mathbf{q})$ is the second-order kernel of standard perturbation theory. A more detailed description of these functions is presented in Crocce et al. [39].

In configuration space, equation (3.5) implies that the non-linear correlation function will be given by an analogous decomposition

$$
\xi_{\mathrm{NL}}(r, z)=\xi_{\mathrm{L}}(r, z) \otimes \tilde{G}^{2}(r, z)+\xi_{\mathrm{MC}}(r, z),
$$

where $\tilde{G}(r, z)$ is the Fourier transform of the non-linear propagator and the symbol $\otimes$ denotes a convolution. The first term in this equation corresponds to the convolution of the linear theory correlation function with a nearly Gaussian kernel, which is responsible for the damping of the acoustic peak. In turn, the contribution of the mode-coupling term at large pair separations can be computed to leading order using equation (3.8), yielding $\xi_{\mathrm{MC}}(r) \propto \xi_{\mathrm{L}}^{\prime}(r) \xi_{\mathrm{L}}^{(1)}(r)$ [28], where $\xi_{\mathrm{L}}^{\prime}(r)$ is the derivative of the linear theory correlation function and $\xi_{\mathrm{L}}^{(1)}(r)$ is given by

$$
\xi_{\mathrm{L}}^{(1)}(r) \equiv \hat{r} \cdot \nabla^{-1} \xi_{\mathrm{L}}(r)=4 \pi \int P_{\mathrm{L}}(k) j_{1}(k r) k \mathrm{~d} k
$$

with $j_{1}(y)$ denoting the spherical Bessel function of order one.

Based on these results, Crocce \& Scoccimarro [28] proposed to model the shape of the galaxy correlation function on large scales using the following parametrization

$$
\xi_{\mathrm{NL}}(r)=b^{2}\left\{\xi_{\mathrm{L}}(r) \otimes \mathrm{e}^{-\left(k_{\star} r\right)^{2}}+A_{\mathrm{MC}} \xi_{\mathrm{L}}^{\prime}(r) \xi_{\mathrm{L}}^{(1)}(r)\right\},
$$

where $b, k_{\star}$ and $A_{\mathrm{MC}}$ are treated as free parameters. An analogous parametrization can be defined for the power spectrum [41]. These parametrizations give an accurate description of the results of 


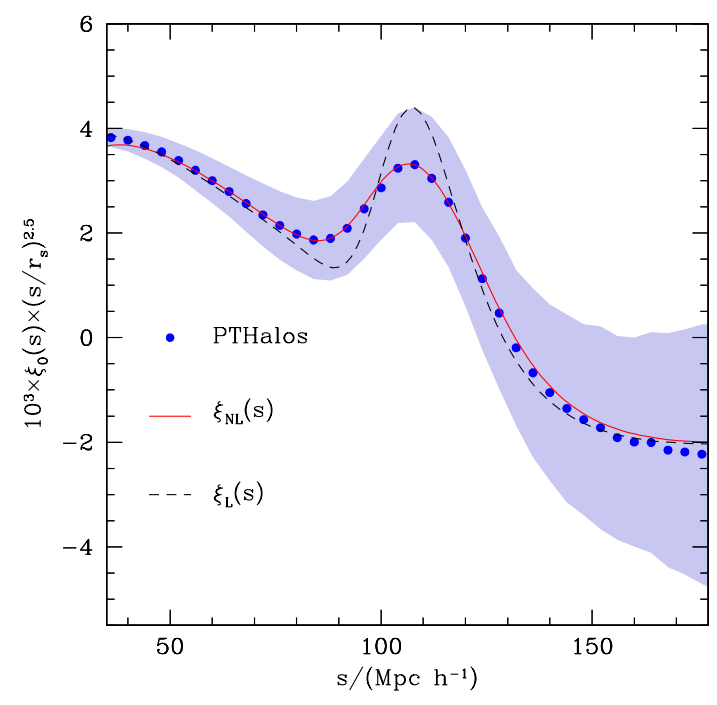

Figure 3: Mean redshift-space two-point correlation function of a set of 600 mock catalogues of the BOSSDR9 CMASS sample [40]. The shaded region corresponds to the variance between the different realizations. The shape of the measured correlation function deviates from the prediction of linear perturbation theory, shown by the dashed line. The solid line corresponds to the parametrization of the galaxy correlation function of equation (3.11), which gives an excellent description of the result obtained from the mock catalogues.

N-body simulations at various redshifts for both real- and redshift-space information [29, 41], and have been used in the analysis of real galaxy clustering measurements [11, 24, 13, 14, 12,

An illustration of how accurately the parametrization of equation (3.11) can reproduce the spatial clustering of true galaxy samples can be seen in Fig. 3. The points correspond to the mean correlation function from an ensemble of 600 mock catalogues of the CMASS sample from BOSS DR9 (see Section \#). To highlight the shape of the acoustic peak, this measurement has been rescaled by $\left(r / r_{s}\right)^{2.5}$, where $r_{\mathrm{s}}=153.2 \mathrm{Mpc}$ corresponds to the sound horizon at the drag redshift for the underlying cosmological model of the mock catalogues. The shaded region corresponds to the variance between the different realizations. This measurement is affected by the same potential problems as real observations: non-linear evolution of density fluctuations, redshift-space distortions, galaxy bias of a sample including both central and satellites, and a non-trivial geometry. Even in this case, the model of equation 3.11, shown by the red solid line, can give an excellent description of the full shape of $\xi(s)$.

\subsection{Reconstruction}

Although the effects of non-linear evolution can be modelled and included in the theoretical description of a given measurement, the damping of the BAO signal leads to a degradation of the achievable accuracy. A complementary approach is to apply corrections to the galaxy positions prior to the estimation of the two-point correlation function or power spectrum to reduce the impact of these systematics. This is the general idea behind the "reconstruction" technique [43].

The dominant source of the damping of the BAO feature is the coherent flows that arise from the clustering pattern itself. This means that the same galaxy sample used to detect the BAO feature 

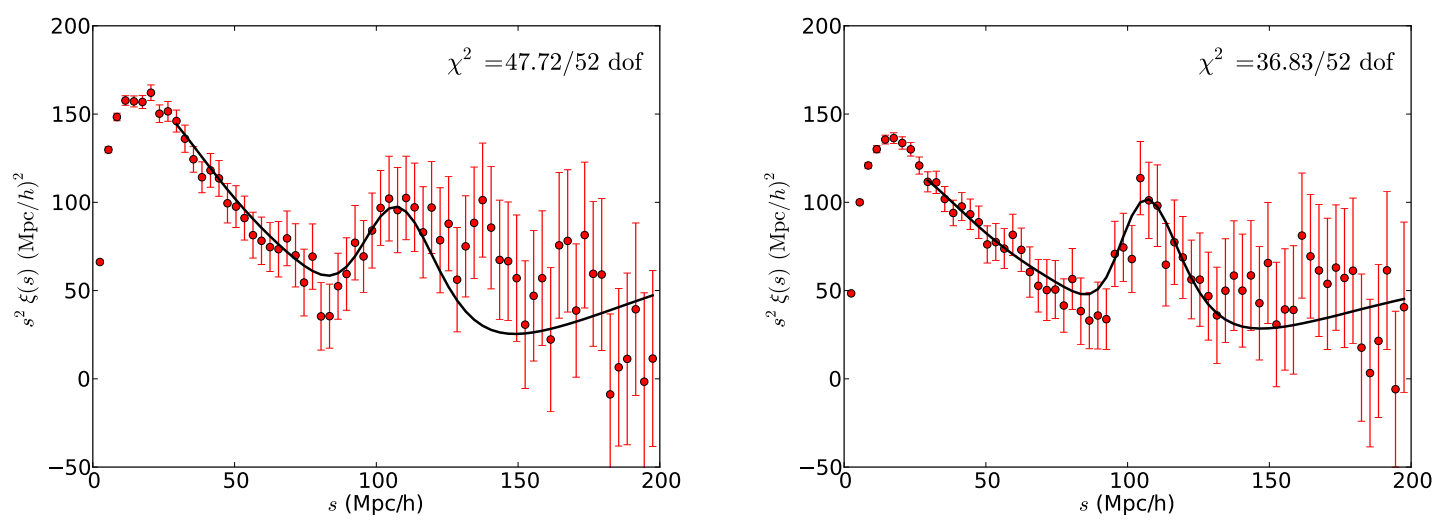

Figure 4: The unreconstructed (left) and reconstructed (right) angle-averaged correlation functions of the LRG sample of SDSS DR7. Reconstruction sharpens the acoustic peak in $\xi(r)$, increasing the significance of its detection. Figure from Padmanabhan et al. [42].

can also map the cosmic structure responsible for its degradation. One can therefore use these data to infer the large-scale flow field and partially undo the damping of the BAO feature.

In practice, reconstruction is performed by computing a displacement field, $\Psi$, based on the Zel'dovich approximation and the description of redshift-space distortions in the linear regime [42]. Assuming a curl-free displacement field, such that $\Psi=-\nabla \phi$, this displacement can be estimated from the measured redshift-space galaxy density field, $\delta_{\mathrm{g}}$, by solving the equation

$$
\nabla^{2} \phi+f \nabla \cdot((\nabla \phi \cdot \hat{\mathbf{s}}) \hat{s})=\frac{\delta_{\mathrm{g}}}{b},
$$

where $\hat{\mathbf{s}}$ corresponds to the line-of-sight direction. Once the displacement field has been obtained, it is used to move the galaxies closer to their initial positions. An illustration of the results of this procedure can be seen in Fig. 1 , which shows the unreconstructed (left) and reconstructed (right) angle-averaged correlation functions of the LRG sample of SDSS DR7 from Padmanabhan et al. [42]. Reconstruction significantly sharpens the acoustic peak, leading to and increasing the significance of its detection from $3.3 \sigma$ in the unreconstructed case to $4.2 \sigma$ after reconstruction.

Although Gaussian density fluctuations can be completely characterized by their two-point statistics, non-linear evolution transfers information to higher orders. Reconstruction partially restores this information back to the two-point statistics of the density field. In this way, this technique makes it possible to extract more information from two-point clustering measurements than what is obtained through their accurate modelling in the non-linear regime. In general however, reconstruction will not be perfect and residual non-linearities must be taken into account by means of accurate models.

\section{Current constraints from LSS observations: The Baryon Oscillation Spectroscopic Survey}

In this section we review the constraints on cosmological parameters attainable using presentday LSS datasets. For this, we use recent results based on the Baryon Oscillation Spectroscopic 


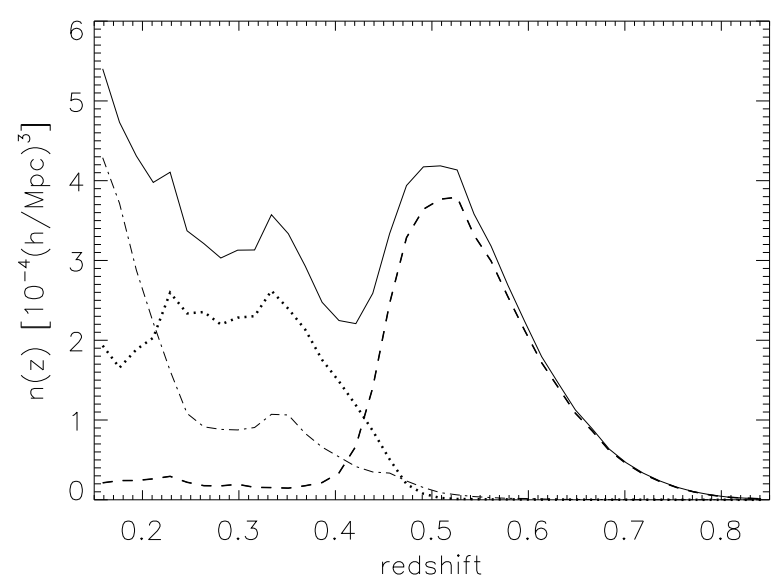

Figure 5: Center: average number density as a function of redshift for the SDSS-II (dash-dotted), LOWZ (dotted), and CMASS DR9 galaxies from BOSS. Figure from Dawson et al. [21].

Survey (BOSS) [21] as an example. BOSS is one of the four components of SDSS-III [44], designed to provide high-precision $\mathrm{BAO}$ measurements at intermediate redshifts $(z \simeq 0.5)$ from largescale galaxy clustering, and at high redshift $(z \simeq 2.5)$ from the Ly $\alpha$ forest signal inferred from a quasar sample. Fig. 5 shows the number density of BOSS galaxies as a function of redshift. BOSS contains two separate galaxy samples, dubbed LOWZ and CMASS, covering different redshift ranges whit a roughly constant number density of $n \simeq 3 \times 10^{-4} h^{3} \mathrm{Mpc}^{-3}$.

The data corresponding to the first two years of observations of BOSS has been publicly released as a part of the SDSS Data Release 9 (DR9) [45]. Even though this sample corresponds to about one third of the final survey, it is already the largest galaxy catalogue available, making it ideally suited for large-scale structure analyses. The first galaxy clustering measurements based on this dataset have shown clear detections of the BAO feature [15]. The circles in the left hand panel of Fig. 6 correspond to the measurement of the correlation function of the CMASS galaxy sample of BOSS-DR9 obtained by Sánchez et al. [12]. The acoustic peak can be clearly seen as a broad bump on large scales. The squares in the same figure correspond to the correlation function of the LRG sample of SDSS-DR3 from Eisenstein et al. [6]. The left-hand side of Fig. 6 shows a similar comparison between the BAO signal in the CMASS power spectrum from Anderson et al. [15] (lower panel) and that of the final LRG sample of SDSS-DR7 [9]. The comparison of these measurements clearly illustrates the improvement in the accuracy of the LSS observations associated with new galaxy surveys like BOSS. Despite the differences in the statistical errors, mean redshifts, and galaxy populations, these measurements show a completely consistent picture of the BAO features in $\xi(r)$ and $P(k)$, indicating the robustness of the results.

The galaxy clustering measurements from BOSS have been used to constrain cosmological parameters [12, 15, 46, 47]. In particular, Sánchez et al. [12] explored the cosmological implications of the full shape of the angle-averaged correlation function using the parametrization described in Section 3.2, combining this information with recent CMB, Type Ia supernova ( $\mathrm{SN}$ ), and additional $\mathrm{BAO}$ measurements from other surveys. An example of the obtained constraints can be seen in 

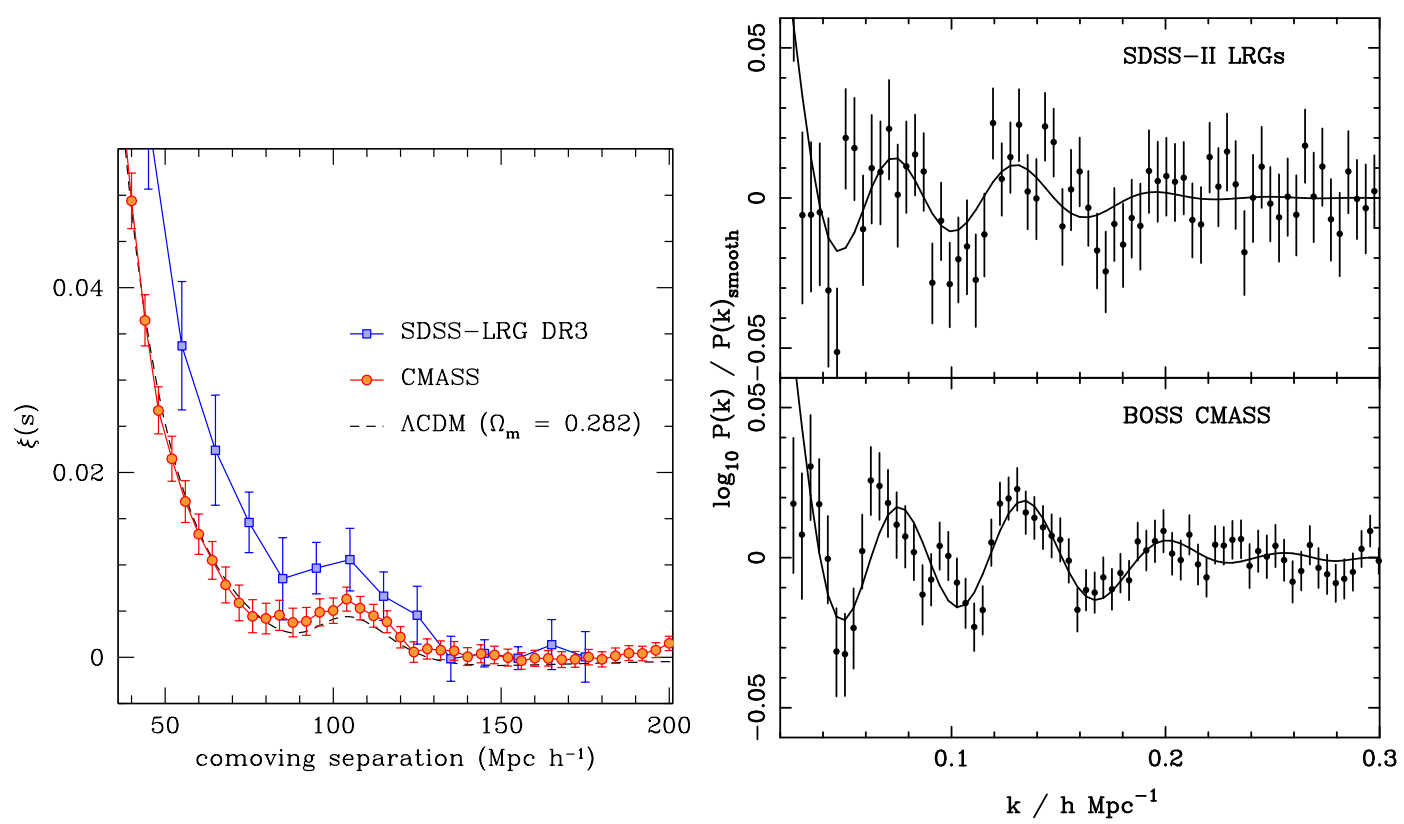

Figure 6: Left-hand sidel: spherically averaged two-point correlation function of the BOSS CMASS sample (circles), compared to the LRG correlation function from Eisenstein et al. [6]. The dashed line corresponds to the best-fitting $\Lambda \mathrm{CDM}$ model to the BOSS data. Right-hand side: BAO signal in the power spectrum of the CMASS sample from BOSS-DR9 (lower panel) compared with that of the final LRG sample of SDSS-II. Figure from Anderson et al. [15].

Fig. 7, which shows the two-dimensional constraints in the $\Omega_{\mathrm{DE}}-w_{\mathrm{DE}}$ plane obtained from different dataset combinations. The CMB-only results (blue dashed lines) exhibit a strong degeneracy which covers a wide range of values of $w_{\mathrm{DE}}$. The solid lines show the effect of including the information of the CMASS two-point correlation function in the analysis. These data alleviate the degeneracy obtained from the $\mathrm{CMB}$ observations, substantially tightening the constraints on the dark energy equation of state. Including also additional $\mathrm{BAO}$ and $\mathrm{SN}$ data in the analysis leads to a final constraint of $w_{\mathrm{DE}}=1.03 \pm 0.07$, in excellent agreement with the standard $\Lambda \mathrm{CDM}$ model value of $w_{\mathrm{DE}}=1$, indicated by a dotted line.

All galaxy clustering measurements from BOSS are in complete agreement with the standard $\Lambda \mathrm{CDM}$ model. This can be seen in Fig.6, where the dashed line corresponds to the prediction for the correlation function in this cosmological model, which gives an excellent match to both the location of the BAO peak and the full shape of the correlation function. This agreement is reinforced when several LSS observations are combined. As discussed in Section 2 , BAO observations can be used to measure the combination $D_{\mathrm{V}}(z) / r_{\mathrm{s}}$. The left panel of Fig. 8 shows the distances inferred from BAO measurements on various spectroscopic data sets [15, 24, 42, 13, rescaled by a fiducial value $r_{\mathrm{s}}^{\mathrm{f}}=153.19$ to express the results in Mpc. The grey region corresponds to the $1 \sigma$ prediction from WMAP assuming a flat $\Lambda$ CDM model. The agreement between these prediction and the various BAO measurements is excellent. A more detailed view of these measurements can be seen in the right panel of Fig. 8, which shows the BAO distance measurements divided by the WMAP $\Lambda$ CDM prediction. The BAO results agree with the best-fitting WMAP model at the few per cent level. The dashed line corresponds to the best-fitting $\Lambda$ CDM model to the combination of WMAP with 


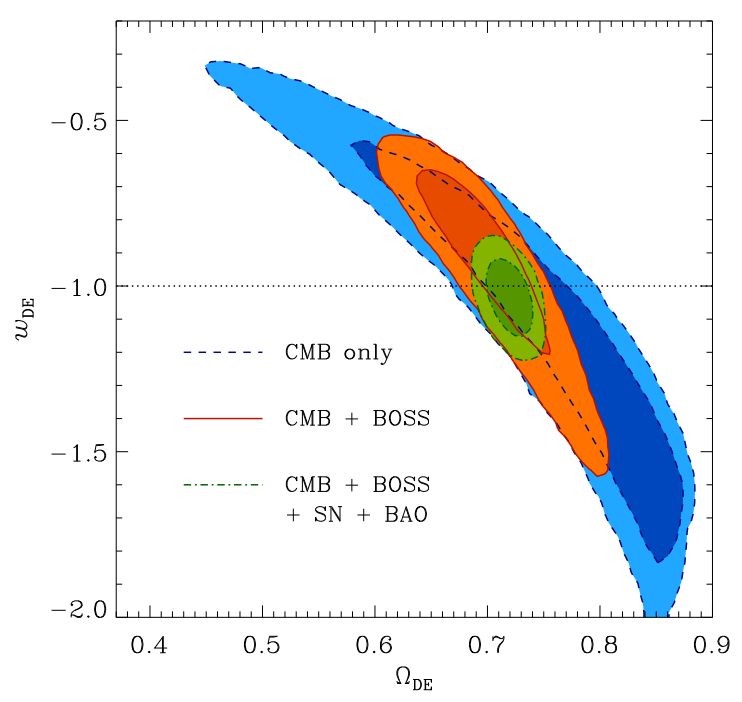

Figure 7: Two-dimensional joint constraints in the $\Omega_{\mathrm{DE}}-w_{\mathrm{DE}}$ plane. The contours show the 68 and 95 per cent confidence levels obtained using CMB data alone (blue dashed lines) and CMB plus the CMASS two-point correlation function (red solid lines). The CMB only constraints show degeneracies which can be reduced by the extra information contained in the shape of the CMASS two-point correlation function. The green dot-dashed lines correspond to the results obtained when the additional BAO and SN information is added to the $\mathrm{CMB}+\mathrm{CMASS}$ combination. These results are in good agreement with the standard $\Lambda \mathrm{CDM}$ value of $w_{\mathrm{DE}}=-1$, indicated by the dotted lines.

the $\mathrm{BAO}$ measurements shown in the figure, which gives an excellent description to all data sets.

\section{5. conclusions}

Current galaxy clustering measurements offer more accurate views of the galaxy clustering pattern than ever before. These observations can play a major role at understanding the origin of cosmic acceleration, but they demand the use of accurate models to avoid introducing systematic errors or biases in the obtained constraints. Although more work needs to be done, the recent developments in the modelling of non-linear evolution of density fluctuations, redshift-space distortions and galaxy bias, as well as the methods to minimize these distortions, provide us with powerful tools to extract cosmological information out of these observations, helping to reach their maximum potential.

Current galaxy clustering measurements can place strong cosmological constraints. These are completely consistent with the standard $\Lambda \mathrm{CDM}$ model, which is able to accurately describe a variety of observations, ranging from SN Type Ia to the CMB. Future galaxy surveys such as Euclid will provide even more accurate views of the large-scale clustering pattern in the Universe. These new surveys will be accompanied by the release of the CMB measurements from the ESA Planck satellite in early 2013. The combination of these datasets will undoubtedly provide new, more stringent constraints on cosmological parameters, putting the $\Lambda \mathrm{CDM}$ model to a much more rigorous test. 

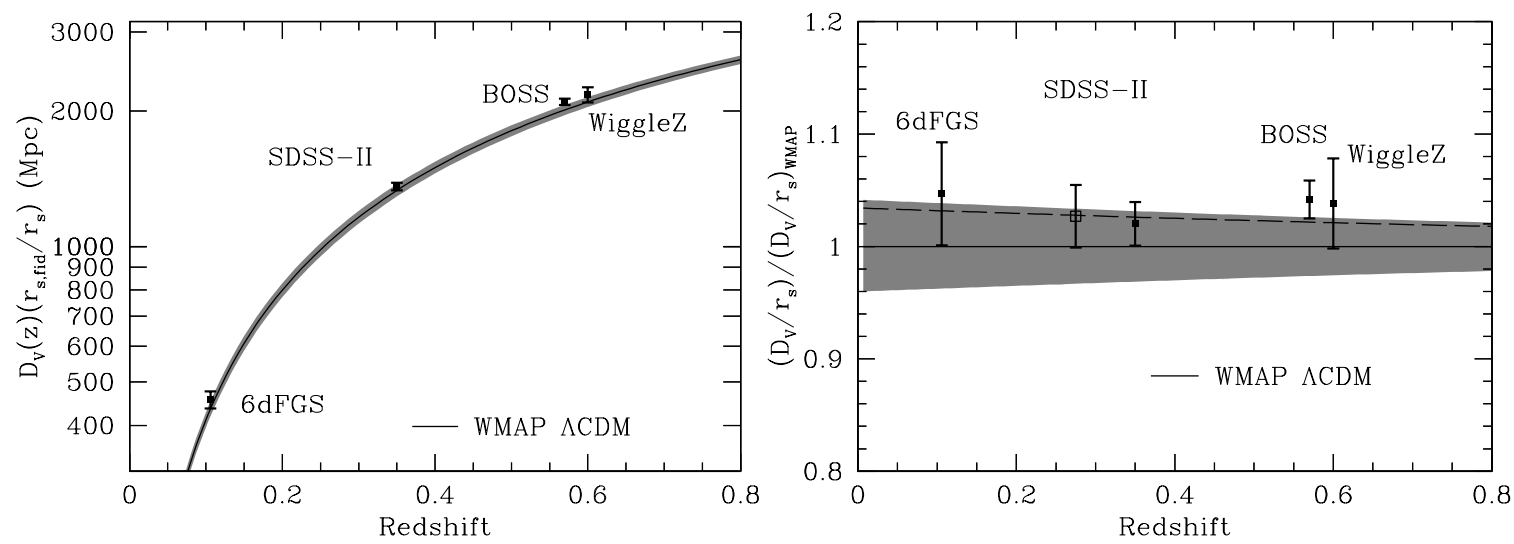

Figure 8: Left-hand side: distances inferred from BAO measurements on various spectroscopic surveys [15], 24, 42, 13]. The grey region corresponds to the $1 \sigma$ prediction from WMAP assuming a flat $\Lambda$ CDM model, which shows a good agreement with the BAO measurements. Right-hand side: BAO distance measurements divided by the WMAP $\Lambda$ CDM prediction. The dashed line corresponds to the best-fitting $\Lambda$ CDM model to the combination of WMAP with the BAO datasets shown in the figure. Figures from Anderson et al. [15]

\section{References}

[1] A. G. Riess, et al., Observational Evidence from Supernovae for an Accelerating Universe and a Cosmological Constant, AJ 116 (Sept., 1998) 1009-1038, [astro-ph/9805201].

[2] S. Perlmutter, et al., Measurements of Omega and Lambda from 42 High-Redshift Supernovae, ApJ 517 (June, 1999) 565-586, astro-ph/9812133.

[3] M. Davis and P. J. E. Peebles, A survey of galaxy redshifts. $V$ - The two-point position and velocity correlations, ApJ 267 (Apr., 1983) 465-482.

[4] G. Efstathiou, et al., Evidence for a non-zero $\Lambda$ and a low matter density from a combined analysis of the 2dF Galaxy Redshift Survey and cosmic microwave background anisotropies, MNRAS 330 (Feb., 2002) L29-L35, astro-ph/0109152.

[5] S. Cole, et al., The 2dF Galaxy Redshift Survey: power-spectrum analysis of the final data set and cosmological implications, MNRAS 362 (Sept., 2005) 505-534, [astro-ph/0501174].

[6] D. J. Eisenstein, et al., Detection of the Baryon Acoustic Peak in the Large-Scale Correlation Function of SDSS Luminous Red Galaxies, ApJ 633 (Nov., 2005) 560-574, astro-ph/0501171.

[7] G. Efstathiou and J. R. Bond, Cosmic confusion: degeneracies among cosmological parameters derived from measurements of microwave background anisotropies, MNRAS 304 (Mar., 1999) 75-97, astro-ph/9807103.

[8] W. J. Percival, et al., Parameter constraints for flat cosmologies from cosmic microwave background and 2dFGRS power spectra, MNRAS 337 (Dec., 2002) 1068-1080, astro-ph/0206256.

[9] W. J. Percival, et al., Baryon acoustic oscillations in the Sloan Digital Sky Survey Data Release 7 galaxy sample, MNRAS 401 (Feb., 2010) 2148-2168, arXiv:0907.1660].

[10] A. G. Sánchez, C. M. Baugh, W. J. Percival, J. A. Peacock, N. D. Padilla, S. Cole, C. S. Frenk, and P. Norberg, Cosmological parameters from cosmic microwave background measurements and the final 2dF Galaxy Redshift Survey power spectrum, MNRAS 366 (Feb., 2006) 189-207, astro-ph/0507583. 
[11] A. G. Sánchez, M. Crocce, A. Cabré, C. M. Baugh, and E. Gaztañaga, Cosmological parameter constraints from SDSS luminous red galaxies: a new treatment of large-scale clustering, MNRAS 400 (Dec., 2009) 1643-1664, arXiv:0901.2570.

[12] A. G. Sánchez, et al., The clustering of galaxies in the SDSS-III Baryon Oscillation Spectroscopic Survey: cosmological implications of the large-scale two-point correlation function, MNRAS $\mathbf{4 2 5}$ (Sept., 2012) 415-437, arXiv:1203.6616].

[13] C. Blake, et al., The WiggleZ Dark Energy Survey: mapping the distance-redshift relation with baryon acoustic oscillations, MNRAS 418 (Dec., 2011) 1707-1724, [arXiv:1108.2635].

[14] F. Montesano, A. G. Sánchez, and S. Phleps, Cosmological implications from the full shape of the large-scale power spectrum of the SDSS DR7 luminous red galaxies, MNRAS 421 (Apr., 2012) 2656-2681, arXiv:1107.4097.

[15] L. Anderson, et al., The clustering of galaxies in the SDSS-III Baryon Oscillation Spectroscopic Survey: baryon acoustic oscillations in the Data Release 9 spectroscopic galaxy sample, MNRAS 427 (Dec., 2012) 3435-3467.

[16] J. Huchra, M. Davis, D. Latham, and J. Tonry, A survey of galaxy redshifts. IV - The data, ApJS 52 (June, 1983) 89-119.

[17] S. A. Shectman, S. D. Landy, A. Oemler, D. L. Tucker, H. Lin, R. P. Kirshner, and P. L. Schechter, The Las Campanas Redshift Survey, ApJ 470 (Oct., 1996) 172, astro-ph/.

[18] M. Colless, et al., The 2dF Galaxy Redshift Survey: spectra and redshifts, MNRAS 328 (Dec., 2001) 1039-1063, astro-ph/0106498.

[19] D. G. York, et al., The Sloan Digital Sky Survey: Technical Summary, AJ 120 (Sept., 2000) 1579-1587, astro-ph/0006396.

[20] M. J. Drinkwater, et al., The WiggleZ Dark Energy Survey: survey design and first data release, MNRAS 401 (Jan., 2010) 1429-1452, arXiv:0911.4246].

[21] K. S. Dawson, et al., The Baryon Oscillation Spectroscopic Survey of SDSS-III, AJ 145 (Jan., 2013) 10, [arXiv:1208.0022].

[22] R. Laureijs, et al., Euclid Definition Study Report, ArXiv e-prints arXiv:1110.3193.

[23] T. Matsubara, Correlation Function in Deep Redshift Space as a Cosmological Probe, ApJ 615 (Nov., 2004) 573-585, astro-ph/0408349.

[24] F. Beutler, et al., The 6dF Galaxy Survey: baryon acoustic oscillations and the local Hubble constant, MNRAS 416 (Oct., 2011) 3017-3032, arXiv:1106.3366.

[25] G. Hinshaw, et al., Nine-Year Wilkinson Microwave Anisotropy Probe (WMAP) Observations: Cosmological Parameter Results, ArXiv e-prints (Dec., 2012) arXiv:1212.5226.

[26] C. Blake and K. Glazebrook, Probing Dark Energy Using Baryonic Oscillations in the Galaxy Power Spectrum as a Cosmological Ruler, ApJ 594 (Sept., 2003) 665-673, astro-ph/0301632.

[27] R. E. Smith, R. Scoccimarro, and R. K. Sheth, Motion of the acoustic peak in the correlation function, Phys. Rev. D 77 (Feb., 2008) 043525, astro-ph/0703620.

[28] M. Crocce and R. Scoccimarro, Nonlinear evolution of baryon acoustic oscillations, Phys. Rev. D 77 (Jan., 2008) 023533, arXiv:0704.2783.

[29] A. G. Sánchez, C. M. Baugh, and R. E. Angulo, What is the best way to measure baryonic acoustic oscillations?, MNRAS 390 (Nov., 2008) 1470-1490, arXiv:0804.0233. 
[30] R. E. Angulo, C. M. Baugh, C. S. Frenk, and C. G. Lacey, The detectability of baryonic acoustic oscillations in future galaxy surveys, MNRAS 383 (Jan., 2008) 755-776, [astro-ph/0 702543].

[31] N. Kaiser, Clustering in real space and in redshift space, MNRAS 227 (July, 1987) 1-21.

[32] R. Scoccimarro, Redshift-space distortions, pairwise velocities, and nonlinearities, Phys. Rev. D 70 (Oct., 2004) 083007, astro-ph/0407214].

[33] H. J. Mo and S. D. M. White, An analytic model for the spatial clustering of dark matter haloes, MNRAS 282 (Sept., 1996) 347-361, [astro-ph/9512127].

[34] R. K. Sheth, H. J. Mo, and G. Tormen, Ellipsoidal collapse and an improved model for the number and spatial distribution of dark matter haloes, MNRAS 323 (May, 2001) 1-12, astro-ph/9907024.

[35] M. Crocce and R. Scoccimarro, Renormalized cosmological perturbation theory, Phys. Rev. D 73 (Mar., 2006) 063519, astro-ph/0509418.

[36] T. Matsubara, Resumming cosmological perturbations via the Lagrangian picture: One-loop results in real space and in redshift space, Phys. Rev. D 77 (Mar., 2008) 063530, [arXiv:0711.2521].

[37] M. Pietroni, Flowing with time: a new approach to non-linear cosmological perturbations, J. Cosmol. Astropart. Phys. 10 (Oct., 2008) 36, arXiv:0806.0971.

[38] A. Taruya and T. Hiramatsu, A Closure Theory for Nonlinear Evolution of Cosmological Power Spectra, ApJ 674 (Feb., 2008) 617-635, [arXiv:0708.1367].

[39] M. Crocce, R. Scoccimarro, and F. Bernardeau, MPTBREEZE: a fast renormalized perturbative scheme, MNRAS 427 (Dec., 2012) 2537-2551, arXiv:1207.1465.

[40] M. Manera, et al., The clustering of galaxies in the SDSS-III Baryon Oscillation Spectroscopic Survey: a large sample of mock galaxy catalogues, ArXiv e-prints (Mar., 2012) arXiv:1203.6609.

[41] F. Montesano, A. G. Sánchez, and S. Phleps, A new model for the full shape of the large-scale power spectrum, MNRAS 408 (Nov., 2010) 2397-2412, arXiv: 1007.0755.

[42] N. Padmanabhan, X. Xu, D. J. Eisenstein, R. Scalzo, A. J. Cuesta, K. T. Mehta, and E. Kazin, A 2 per cent distance to $z=0.35$ by reconstructing baryon acoustic oscillations - I. Methods and application to the Sloan Digital Sky Survey, MNRAS 427 (Dec., 2012) 2132-2145, [arXiv:1202.0090].

[43] D. J. Eisenstein, H.-J. Seo, E. Sirko, and D. N. Spergel, Improving Cosmological Distance Measurements by Reconstruction of the Baryon Acoustic Peak, ApJ 664 (Aug., 2007) 675-679, astro-ph/0604362].

[44] D. J. Eisenstein, et al., SDSS-III: Massive Spectroscopic Surveys of the Distant Universe, the Milky Way, and Extra-Solar Planetary Systems, AJ 142 (Sept., 2011) 72, [arXiv:1101.1529].

[45] C. P. Ahn, et al., The Ninth Data Release of the Sloan Digital Sky Survey: First Spectroscopic Data from the SDSS-III Baryon Oscillation Spectroscopic Survey, ApJS 203 (Dec., 2012) 21, arXiv:1207.7137].

[46] B. A. Reid, et al., The clustering of galaxies in the SDSS-III Baryon Oscillation Spectroscopic Survey: measurements of the growth of structure and expansion rate at $z=0.57$ from anisotropic clustering, MNRAS 426 (Nov., 2012) 2719-2737, arXiv:1203.6641].

[47] L. Samushia, et al., The clustering of galaxies in the SDSS-III DR9 Baryon Oscillation Spectroscopic Survey: testing deviations from $\Lambda$ and general relativity using anisotropic clustering of galaxies, MNRAS 429 (Feb., 2013) 1514-1528, [arXiv:1206.5309]. 